\title{
Pathogens distribution and antimicrobial resistance in bloodstream infections in twenty-five neonatal intensive care units in China, 2017-2019
}

Jing Liu' ${ }^{1}$ Zengyu Fang ${ }^{1}$, Yonghui Yu ${ }^{1,2^{*}}$, Yanjie Ding ${ }^{3}$, Zhijie Liư ${ }^{4}$, Chengyuan Zhang ${ }^{5}$, Haiying He ${ }^{6}$, Hongli Geng ${ }^{7}$, Weibing Chen ${ }^{8}$, Guoying Zhao ${ }^{9}$, Qiang Liu ${ }^{10}$, Baoying Wang ${ }^{11}$, Xueming Sun ${ }^{12}$, Shaofeng Wang ${ }^{13}$, Rongrong Sun ${ }^{14}$, Delong Fu ${ }^{15}$, Xinjian Liu ${ }^{16}$, Lei Huang ${ }^{17}$, Jing Li ${ }^{18}$, Xuexue Xing ${ }^{19}$, Xiaokang Wang ${ }^{20}$, Yanling Gao ${ }^{21}$, Renxia Zhu ${ }^{22}$, Meiying Han ${ }^{23}$, Fudong Peng ${ }^{24}$, Min Geng ${ }^{25}$ and Liping Deng ${ }^{26}$

\begin{abstract}
Background: Overcrowding, abuse of antibiotics and increasing antimicrobial resistance negatively affect neonatal survival rates in developing countries. We aimed to define pathogens and their antimicrobial resistance (AMR) of early-onset sepsis (EOS), hospital-acquired late-onset sepsis (HALOS) and community-acquired late-onset sepsis (CALOS) in 25 neonatal intensive care units (NICUs) in China.
\end{abstract}

Study design: This retrospective descriptive study included pathogens and their AMR from all neonates with bloodstream infections (BSIs) admitted to 25 tertiary hospitals in China from January 1, 2017, and December 31, 2019. We defined EOS as the occurrence of BSI at or before $72 \mathrm{~h}$ of life and late-onset sepsis (LOS) if BSI occurred after $72 \mathrm{~h}$ of life. LOS were classified as CALOS if occurrence of BSI was $\leq 48 \mathrm{~h}$ after admission, and HALOS, if occurrence was $>48 \mathrm{~h}$ after admission.

Results: We identified 1092 pathogens of BSIs in 1088 infants from 25 NICUs. Thirty-two percent of all pathogens were responsible for EOS, 64.3\% HALOS, and 3.7\% CALOS. Gram-negative (GN) bacteria accounted for a majority of pathogens in EOS (56.7\%) and HALOS (62.2\%). The most frequent pathogens causing EOS were Escherichia coli (27.2\%) and group B streptococcus (GBS; 14.6\%) whereas in CALOS they were GBS (46.3\%) and Staphylococcus aureus (41.5\%). Klebsiella pneumoniae (27.9\%), Escherichia coli (15.7\%) and Fungi (12.8\%) were the top three isolates in HALOS. Third-generation cephalosporin resistance rates in GN bacteria ranged from 9.7 to $55.6 \%$ in EOS and 26\% to 63.3\% in HALOS. Carbapenem resistance rates in GN bacteria ranged from 2.7 to $31.3 \%$ in HALOS and only six isolates in EOS were carbapenem resistant. High rates of multidrug resistance were observed in Klebsiella pneumoniae (60.7\%) in HALOS and in Escherichia coli (44.4\%) in EOS. All gram-positive bacteria were susceptible to vancomycin except for three Enterococcus faecalis in HALOS. All-cause mortality was higher among neonates with EOS than HALOS (7.4\% VS 4.4\%, [OR] 0.577, 95\% Cl 0.337-0.989; $P=0.045)$.

*Correspondence: alice20402@126.com

1 Department of Neonatology, Shandong Provincial Hospital Affiliated to Shandong First Medical University, Jinan 250021, Shandong, China

Full list of author information is available at the end of the article permits use, sharing, adaptation, distribution and reproduction in any medium or format, as long as you give appropriate credit to the original author(s) and the source, provide a link to the Creative Commons licence, and indicate if changes were made. The images or other third party material in this article are included in the article's Creative Commons licence, unless indicated otherwise in a credit line to the material. If material is not included in the article's Creative Commons licence and your intended use is not permitted by statutory regulation or exceeds the permitted use, you will need to obtain permission directly from the copyright holder. To view a copy of this licence, visit http://creativecommons.org/licenses/by/4.0/. The Creative Commons Public Domain Dedication waiver (http://creativeco mmons.org/publicdomain/zero/1.0/) applies to the data made available in this article, unless otherwise stated in a credit line to the data. 
Conclusions: Escherichia coli, Klebsiella pneumoniae and GBS were the leading pathogens in EOS, HALOS and CALOS, respectively. The high proportion of pathogens and high degree of antimicrobial resistance in HALOS underscore understanding of the pathogenesis and emphasise the need to devise effective interventions in developing countries.

Keywords: Neonatal sepsis, Antimicrobial resistance, Neonatal intensive care unit, Klebsiella pneumoniae

\section{Background}

Neonatal bloodstream infection (BSI) is the third most common cause of neonatal morbidity and mortality globally, and is an ongoing major global public health challenge $[1,2]$. Asia and Africa have the highest burden of BSIs in the world [2]. Scarcity in resources, insufficient surveillance and infection control, abuse of antibiotics and increase of antimicrobial resistance in low-income and middle-income countries (LMICs) may contribute to this situation [3, 4]. The risk of emergence and spread of antibiotic resistance in South East Asia is thought to be among the highest among all the WHO regions [5, 6]. Monitoring resistance in disease causing pathogens is of particular importance for neonatal BSIs in LMICs, where most treatments are empirically prescribed but should be based on reliable contemporaneous resistance data.

Neonatal sepsis was classified into early onset sepsis (EOS) and late onset sepsis (LOS) routinely $[7,8]$. EOS generally reflects vertical transmission from mothers while LOS cases were likely due to pathogens acquired after delivery and often from nosocomial infections [9]. A previous meta-analysis reported that Staphylococcus species, especially Coagulase negative Staphylococcus (CoNS) continue to be the principal organisms of neonatal sepsis in China [10]. Nevertheless, more recent data described Klebsiella pneumoniae as the most frequent pathogen, with widespread antimicrobial resistance (AMR) [11]. Klebsiella pneumoniae was primarily associated with LOS, greater morbidity, mortality and limited treatment options in neonates $[11,12]$. In China, multicenter reports on pathogens of neonatal BSI were scarce and were limited to specific gestational age groups or not involving resistance analysis of antibiotics [12,13]. Currently, data on antimicrobial resistance (AMR) distinguishing between community-acquired LOS (CALOS) and hospitalacquired LOS (HALOS) in neonates are also scarce [3, 14]. Detecting emerging resistance in neonatal BSI is vital in order to optimise empiric antibiotic therapy in HALOS, in accordance with antimicrobial stewardship principle and to reduce mortality. The present study assessed the benchmark in neonatal sepsis, distinguishing between EOS, HALOS, and CALOS, and covering the neonatal population of entire gestational age groups.

\section{Methods}

\section{Settings and infection control methods}

Twenty-five tertiary hospitals participated in the current study. Among the hospitals, twenty-three tertiary hospitals were located in Shandong province which involving 13 major cities, one tertiary hospital in Hebei province and the other one in the Inner Mongolia Autonomous Region. Nineteen hospitals were general hospitals and 6 were maternal and child health care hospitals.

Because all 25 hospitals have their own maternity/ obstetric ward, most neonates were born on site and only a few were transferred. The number of beds ranged from 20 to 60 . The ratio of nurses to bed ranged from 0.4 to 1.2 and physician to nurse ranged from 0.3 to 0.5 . The bed occupancy rate was maintained above $90 \%$. All 25 hospitals have an infection control committee. Trained infection control nurses were available at all units at all times. All NICUs had a hand hygiene policy, but no audits of staff compliance were undertaken. Alcohol-based hand rub solutions and disinfectant dispensers filled with betadine $7.5 \%$ were provided at hand-wash sinks, and clean disposable tissue papers for hand-drying were sufficiently available. None of 25 NICUs had laminar flow devices. Surveillance cultures were only used when an outbreak was suspected but were not routinely undertaken.

\section{Identification and susceptibility testing}

Blood cultures were performed for any infant presenting with clinical signs or symptoms of sepsis according to the local guidelines of each hospital. Blood samples were collected by trained nurses or physicians. Venipuncture sites were prepared with $75 \%$ isopropyl alcohol, followed by iodine tincture, and then wiped with alcohol. Skin site was allowed to dry for 1 min prior to venipuncture. A general policy of using one culture bottle exclusively for newborns with at least $1 \mathrm{ml}$ of blood sample was adopted by all hospitals. The sample was delivered to the microbiology laboratory within $2 \mathrm{~h}$ of collection by staff members. Training of blood culture collection procedures were undertaken regularly in local hospitals. Each microbiology laboratory performed routine microbiology tests, including organism identification and antimicrobial susceptibility testing (AST). Blood cultures were performed at recruited hospital laboratories and incubated using Bactec FX system (Becton Dickinson, USA) in 15 hospitals and BacT/ALERT 3D system (bioMérieux, 
France) in 10 hospitals. Automated methods include use of VITEK-2 compact system in 23 hospitals and VitekMS system in 2 hospitals for organism identification and AST. Manual methods include organism identification by agar plate and biochemical workup and AST by disk diffusion methods. AST of pathogens was undertaken according to Clinical and Laboratory Standards Institute guidelines $[15,16]$.

\section{Definitions}

Inclusion criteria of infants: infants gestational age $\geq 37$ weeks with sepsis occurred within 28 days after birth and infants gestational age $<37$ weeks with sepsis occurred within the corrected age of 44 weeks $[14,17$, $18]$.

Our definition of neonatal sepsis was formulated with consideration to Chinese consensus of diagnosis and treatment. Neonatal sepsis was defined as the growth of at least a single pathogen (bacterium or fungus) from the blood of an infant who fulfilled all three of the following criteria: (1) One or more of the following infection-related clinical manifestations: respiratory distress, apnea; tachycardia or bradycardia; systemic hypotension or hypoperfusion; hypothermia or fever $\left(\mathrm{T}>38.5^{\circ} \mathrm{C}\right.$ or $<36^{\circ} \mathrm{C}$ ); convulsions, hypotonia, irritability or lethargy; feeding intolerance or intestinal obstruction. (2) One or more abnormal hematologic index: white blood cell count $\left(<5 \times 10^{\wedge} 9 / \mathrm{L}\right.$ or $>30 \times 10^{\wedge} 9 / \mathrm{L}$ for age $\leq 3 \mathrm{~d}$ or $>20 \times 10^{\wedge} 9 / \mathrm{L}$ for age $>3 \mathrm{~d}$ ), increase of immature/total neutrophil $\left(\geq 0.16\right.$ for age $<3 \mathrm{~d}$ or $\geq 0.12 \times 10^{\wedge} 9 / \mathrm{L}$ for age $\geq 3 \mathrm{~d}$ ), C-reactive protein level $(\geq 10 \mathrm{mg} / \mathrm{L})$ or abnormal procalcitonin level ( $\geq 0.5 \mathrm{mg} / \mathrm{L})$. (3) Antibiotics used for at least 5 days [19-21].

Contaminants were defined based on the following criteria: (1) isolates usually considered as contaminants (eg, Micrococcus species); (2) CoNS in the absence of a peripheral or central catheter when the blood samples was collected; (3) a mixed flora of CoNS was cultured; (4) isolates considered as contaminants by the neonatologist, implying that antibiotics used less than 5 days [14].

EOS was defined as the occurrence of sepsis at or before the first $72 \mathrm{~h}$ of life while LOS was defined as the occurrence of sepsis after the first $72 \mathrm{~h}$ of life. Among LOS, infants with sepsis onset $\leq 48 \mathrm{~h}$ after admission were considered as having CALOS, and those with onset $>48 \mathrm{~h}$ after admission were considered as having HALOS [14].

Repeatedly isolated pathogens were regarded as identical BSI episodes unless they occurred beyond 7 days after the last positive culture result [22]. Antimicrobial susceptibilities were reported as susceptible or resistant (intermediate or resistant) based on microbiology reports. Resistance proportions were reported as number of resistant pathogens/number of pathogens tested.

Multidrug-resistant (MDR) gram-negative (GN) bacteria were defined isolates tested against at least 1 agent in 3 or more of the following antimicrobial categories: carbapenems (imipenem and meropenem), penicillins (piperacillin, Ampicillin, and piperacillin/ tazobactam), broad-spectrum cephalosporins (ceftazidime and cefepime), monobactams (aztreonam), aminoglycosides, and fluoroquinolones [23].

All-cause mortality was defined as a proportion of neonates deceased among admitted neonates [24].

\section{Data collection and statistical analyses}

This study is a retrospective, multicenter case series of hospitalized neonates with positive blood cultures. The medical record of each infant with positive blood culture was reviewed by a local neonatologist and the data was recorded onto a unified standardized worksheet from all 25 NICUs. Data of the worksheet included the medical institution, number of cots, staffing ratios, medical record number, gestational age, birth weight, gender, date of birth, date of blood cultures obtained, isolates identified, clinical significance of isolates and antimicrobial susceptibility. Other clinical data were also collected including body temperature, heart rate, white blood cell count, procalcitonin and C-reactive protein in the $72 \mathrm{~h}$ before and after blood cultures were collected. Worksheets from 25 NICUs were sorted out critically by a neonatologist and a clinical microbiologist was involved in the interpretation of these microbial results. The ethics committees of all 25 participating hospitals approved the study and allowed data sharing. Procedures were in accordance with the Helsinki Declaration of the World Medical Association.

Statistical analysis was performed using the SPSS software version 25.0 (SPSS Inc, Chicago, IL). Descriptive analysis was performed to characterize the study population and pathogens. Categorical data are presented as percentages, numerical data as median with 25th and 75th percentiles (interquartile range, IQR). The univariable logistic regression was used to evaluate group differences in all-cause mortality and fungal BSI. Two-sided $P<0.05$ indicated significance.

\section{Results}

A total of 2752 isolates from 2693 infants were obtained from 25 NICUs between January 1, 2017 and December 31 , 2019. However, only 39.7\% (1092/2752) of isolates were classified as disease causing pathogens that met inclusion criteria, excluding 1644 (59.8\%) contaminants and $16(0.5 \%)$ repeated pathogens (Fig. 1). Of these 1092 pathogens, 349 (32\%) pathogens were responsible for EOS, $702(64.3 \%)$ for HALOS and 41 (3.7\%) for CALOS. 


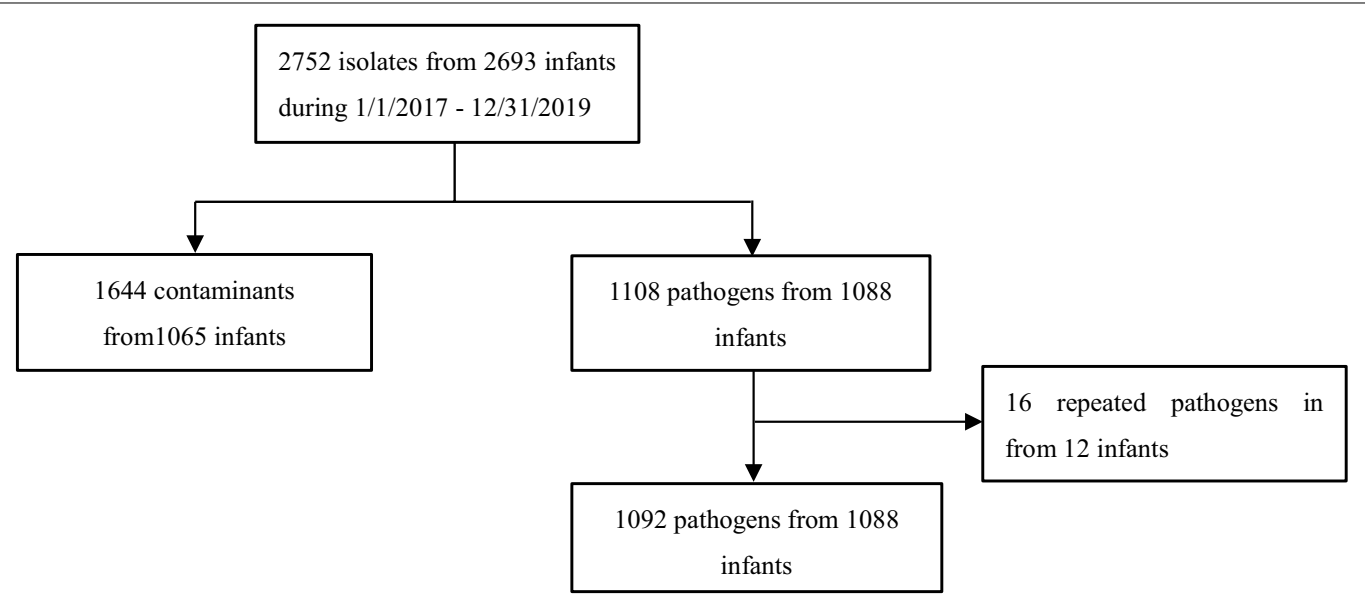

Fig. 1 Study flow diagram

No neonate experienced both EOS and HALOS. Four infants had Klebsiella pneumoniae caused by polymicrobial pathogens, namely one with Escherichia coli and Klebsiella pneumoniae, one with Klebsiella pneumoniae and Enterococcus species, and two infants with Pseudomonas aeruginosa and Klebsiella pneumoniae. The characteristics of the study population are presented in Table 1 . Of the infants studied, $57.4 \%(624 / 1088)$ were male. All-cause mortality was $7.4 \%(26 / 349)$ in EOS and $4.4 \%(31 / 698)$ in HALOS. No infants with CALOS died. All-cause mortality was higher among neonates with EOS than HALOS (7.4\% vs. 4.4\%, [OR] 0.577, 95\% CI $0.337-0.989 ; P=0.045)$. Table 2 shows the pathogen distribution that caused neonatal EOS, HALOS and CALOS. GN bacteria was the commonest in both EOS and HALOS, with the proportion of 56.7\% (198/349) and $62.2 \%$ (437/702), respectively.

\section{Early-onset sepsis}

In EOS, 51\% (178/349) were term infants and 58.2\% $(203 / 349)$ were neonates with normal birth weight. Overall, Escherichia coli and GBS were the most common pathogenic bacteria of EOS, accounting for $27.2 \%$ $(95 / 349)$ and $14.6 \%(51 / 349)$. $84.3 \%(43 / 51)$ of $G B S$ were identified from term infants and $15.7 \%(8 / 51)$ in preterm infants with EOS. In contrast, Escherichia coli were responsible for $61.1 \%$ (58/95) of pathogens in preterm infants, and $38.9 \%$ (37/95) in term infants with EOS. In maternal and child health hospital, Escherichia coli $(19.4 \%$; $24 / 124)$ and Klebsiella pneumoniae $(16.1 \%$;

Table 1 Demographic and clinical characteristics of the study population

\begin{tabular}{|c|c|c|c|c|}
\hline & $\begin{array}{l}\text { All patients } \\
\text { ( } n=1088 \text { infants) }\end{array}$ & $\begin{array}{l}\text { EOS } \\
(n=349 \text { infants })\end{array}$ & $\begin{array}{l}\text { HALOS } \\
(n=698 \text { infants) }\end{array}$ & $\begin{array}{l}\text { CALOS } \\
\text { ( } n=41 \text { infants) }\end{array}$ \\
\hline \multicolumn{5}{|l|}{ Birth weight (grams), n (\%) } \\
\hline$<1500$ & $352(32.4)$ & $65(18.6)$ & $287(41.1)$ & 0 \\
\hline $1501-2500$ & $230(21.1)$ & $81(23.2)$ & $148(21.2)$ & $1(2.4)$ \\
\hline$\geq 2500$ & $506(46.5)$ & $203(58.2)$ & $263(37.7)$ & $40(97.6)$ \\
\hline \multicolumn{5}{|l|}{ Gestational age (weeks), n (\%) } \\
\hline$<28$ & $104(9.6)$ & $23(6.6)$ & $81(11.6)$ & 0 \\
\hline $28-34$ & $386(35.5)$ & $87(24.9)$ & $299(42.8)$ & 0 \\
\hline $34-37$ & $130(11.9)$ & $61(17.5)$ & $65(9.3)$ & $4(9.8)$ \\
\hline$\geq 37$ & $468(43)$ & $178(51)$ & $253(36.2)$ & $37(90.2)$ \\
\hline Male sex, n (\%) & $624(57.4)$ & $184(52.7)$ & $441(63.2)$ & $29(70.7)$ \\
\hline Age during blood sampling (days) (median, IQR) & $10(2-22)$ & $1(0-2)$ & $17(10-27)$ & $13(9-23)$ \\
\hline Length of hospital stay (days) (median, IQR) & $36(15-56)$ & $17(10-36)$ & $39(19-63)$ & $16(11-24)$ \\
\hline All-cause mortality (\%) & $57(5.3)$ & $26(7.4)$ & $31(4.4)$ & 0 \\
\hline
\end{tabular}

IQR interquartile range 
Table 2 Pathogen Distributions in EOS, CALOS and HALOS at 25 NICUs, January 2017-December 2019

\begin{tabular}{|c|c|c|c|c|}
\hline Pathogens & $\begin{array}{l}\text { EOS } \\
(n=349 \text { in } 349 \text { infants) } \\
n(\%)\end{array}$ & $\begin{array}{l}\text { HALOS* } \\
(n=702 \text { in } 698 \text { infants }) \\
n(\%)\end{array}$ & $\begin{array}{l}\text { CALOS } \\
(n=41 \text { in } 41 \text { infants) } \\
n(\%)\end{array}$ & $\begin{array}{l}\text { Total } \\
(\mathrm{n}=1092 \text { in } \\
1088 \text { infants } \\
\mathrm{n}(\%)\end{array}$ \\
\hline Gram-positive bacteria & $149(42.7)$ & $175(25.0)$ & $36(87.8)$ & $360(33.0)$ \\
\hline CONS & $28(8.0)$ & $81(11.5)$ & 0 & $109(10.0)$ \\
\hline GBS & $51(14.6)$ & $15(2.1)$ & 19(46.3) & $85(7.8)$ \\
\hline Staphylococcus aureus & $19(5.4)$ & $35(5.0)$ & $17(41.5)$ & $71(6.6)$ \\
\hline Enterococcus spp. & $11(3.2)$ & $18(2.6)$ & 0 & $29(2.7)$ \\
\hline Listeria monocytogenes & $22(6.3)$ & $1(0.1)$ & 0 & $23(2.1)$ \\
\hline Other Gram-positive bacteria & $18(5.2)$ & 25(3.6) & 0 & $43(4.0)$ \\
\hline Gram-negative bacteria & $198(56.7)$ & $437(62.2)$ & $5(12.2)$ & $640(58.6)$ \\
\hline Escherichia coli & $95(27.2)$ & 110(15.7) & $4(9.8)$ & $209(19.1)$ \\
\hline Klebsiella pneumoniae & $31(8.9)$ & $196(27.9)$ & 0 & $227(20.8)$ \\
\hline Enterobacter spp. & $19(5.4)$ & $50(7.1)$ & $1(2.4)$ & $70(6.5)$ \\
\hline Serratia marcescens & $19(5.4)$ & $19(2.7)$ & 0 & $38(3.5)$ \\
\hline Acinetobacter baumannii & $9(2.6)$ & $28(4.0)$ & 0 & $37(3.4)$ \\
\hline Pseudomonas aeruginosa & $5(1.4)$ & $16(2.3)$ & 0 & $21(2.0)$ \\
\hline Other Gram-negative bacteria & $20(5.8)$ & $18(2.6)$ & 0 & $38(3.5)$ \\
\hline Fungi & $2(0.6)$ & $90(12.8)$ & 0 & $92(8.4)$ \\
\hline Candida albicans & $2(0.6)$ & $37(5.3)$ & 0 & $39(3.6)$ \\
\hline Candida parapsilosis & 0 & $18(2.6)$ & 0 & $18(1.6)$ \\
\hline Candida glabrata & 0 & $10(1.4)$ & 0 & $10(0.9)$ \\
\hline Candida guilliemondii & 0 & $8(1.1)$ & 0 & $8(0.7)$ \\
\hline Candida tropicalis & 0 & $3(0.4)$ & 0 & $3(0.3)$ \\
\hline Otherfungi & 0 & $14(2.0)$ & 0 & $14(1.3)$ \\
\hline
\end{tabular}

*Four infants had HALOS caused by polymicrobial pathogens, namely one with Escherichia coli and Klebsiella pneumoniae, one with Klebsiella pneumoniae and Enterococcus species, and two infants with Pseudomonas aeruginosa and Klebsiella pneumoniae

20/124) were the most common pathogenic bacteria of EOS, followed by GBS $(10.5 \% ; 13 / 124)$. In contrast, the top three pathogens in general hospital were Escherichia coli $(31.6 \% ; 71 / 225)$, GBS $(16.9 \% ; 38 / 225)$ and Listeria monocytogenes (7.1\%; 16/225). Carbapenem resistance was uncommon in EOS: 44.4\% (4/9) of Acinetobacter baumannii isolates and 2.1\% (2/95) of Escherichia coli were resistant (Fig. 2). The proportion of resistant isolates was highest for Escherichia coli in EOS: 84.9\% (79/93) were ampicillin resistant, 49.5\% (47/95) were third-generation cephalosporins resistant and $44.4 \%$ (42/95) were multidrug resistant (Fig. 2).

\section{Hospital-acquired LOS}

Pathogens responsible for HALOS were more common in neonates with gestational age 28-34 weeks (42.8\%; $299 / 698)$, followed by $>37$ weeks $(36.2 \%$; 253/698). Data from HALOS patients noted 41.4\% (287/698) were very low birth weight $(<1500 \mathrm{~g})$ neonates and $37.7 \%$ (263/698) neonates with normal birth weight. Klebsiella pneumoniae, the leading pathogen of HALOS, was responsible for $27.9 \%(196 / 702)$ of the cases, followed by Escherichia coli $(15.7 \%, 110 / 702)$ and Fungi (12.8\%, 90/702). Klebsiella pneumoniae was primarily identified among preterm infants $(75.5 \% ; 148 / 196)$. Klebsiella pneumonia was the top common pathogen both in general hospital $(23 \% ; 121 / 527)$ and in maternal and child health hospital $(42.9 \% ; 75 / 175)$. Escherichia coli (17.3\%; 91/527) and Fungi (14.2\%; 75/527) were the second and third common pathogen in general hospital respectively, whereas CoNS (14.3\%; 25/175) and Escherichia coli $(10.9 \% ; 19 / 175)$ was in a second and third place in maternal and child health hospital. Most GN bacteria in HALOS showed a high degree of antimicrobial resistance, not only to commonly used ampicillins (87.5-100\%) and third-generation cephalosporins (26-63.3\%) but also to reserved antibiotics such as carbapenems (2.7-31.3\%) (Fig. 2). A high proportion of Klebsiella pneumoniae (60.7\%; 119/196), Escherichia coli (37.3\%; 41/110), Pseudomonas aeruginosa (37.5\%; 6/16) and Acinetobacter baumannii (35.7\%; 10/28) in HALOS were multidrug resistant. An outbreak of four carbapenem-resistant Klebsiella pneumoniae strains was presented in a general hospital without further 
A

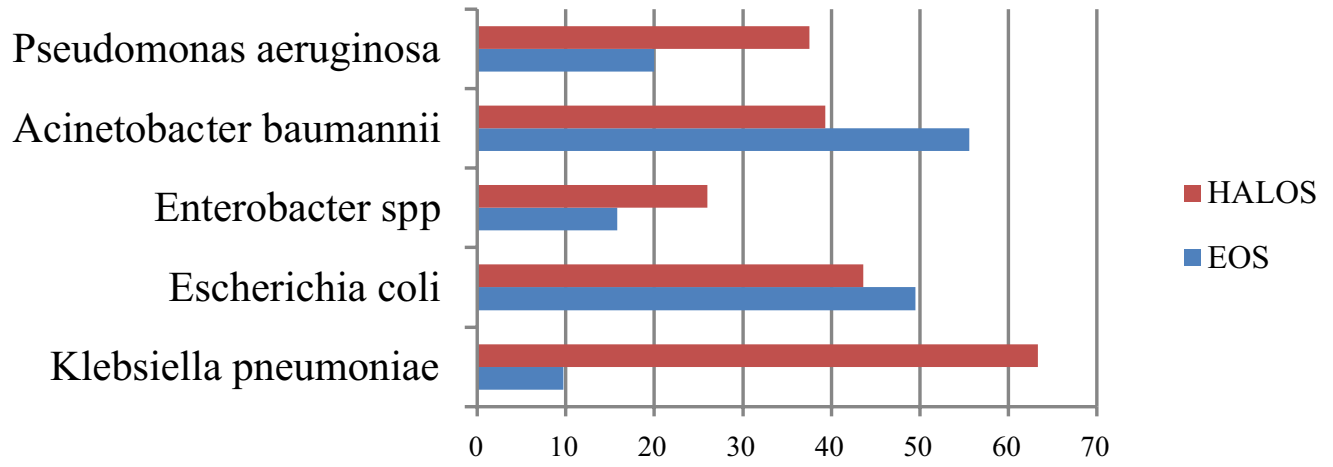

Proportion of third-generation cephalosporin resistance (\%)

B

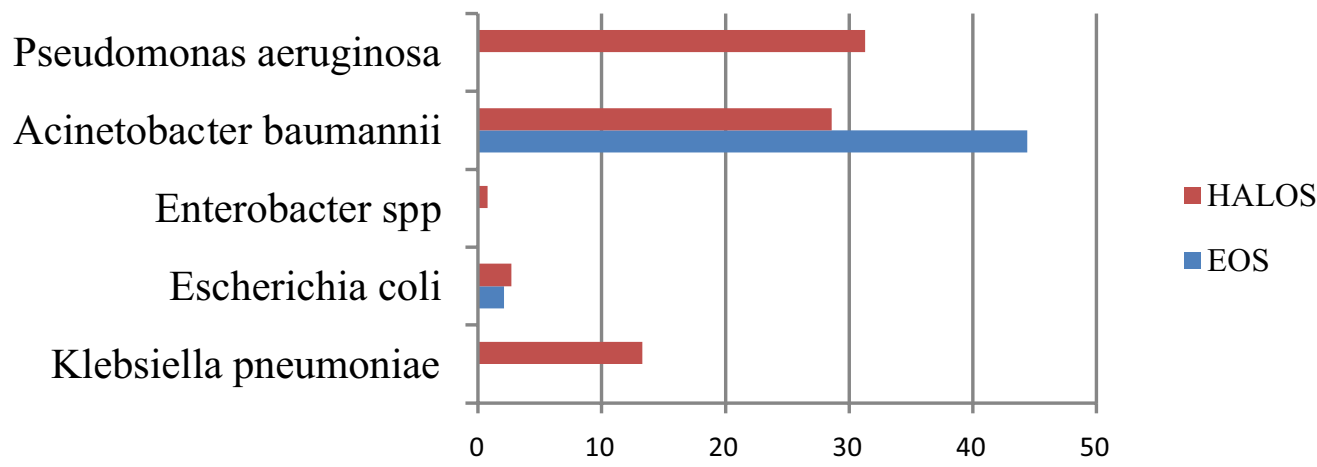

Proportion of carbapenem resistance (\%)

C

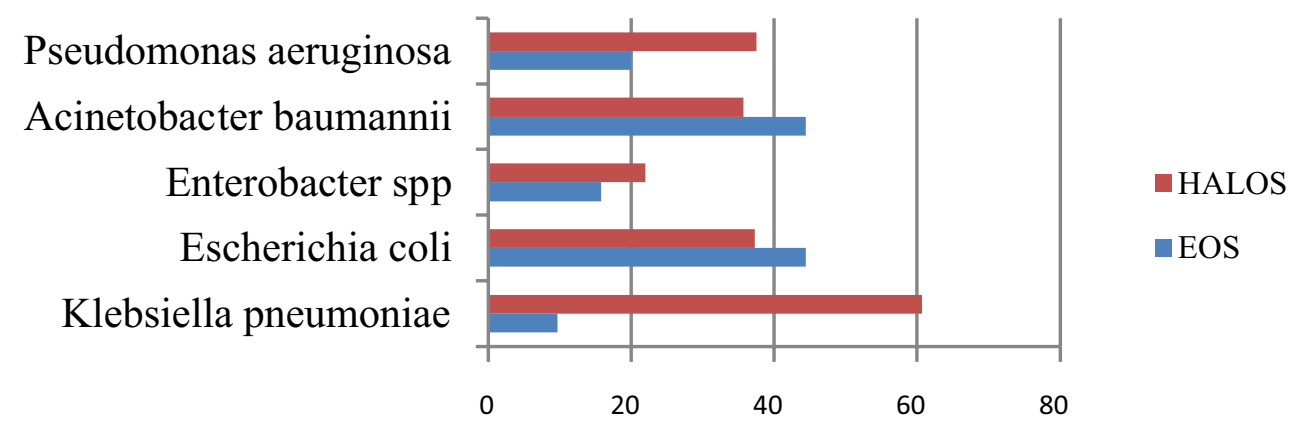

Proportion of multi-drug resistance (\%)

Fig. 2 A Proportion of third-generation cephalosporin resistant main GN bacteria in EOS and HALOS. B Proportion of carbapenem resistant main GN bacteria in EOS and HALOS. C Proportion of multi-drug resistant main GN bacteria in EOS and HALOS;

molecular typing. Among gram-positive (GP) bacteria in HALOS, methicillin resistance was detected in $77.8 \%$ (63/81) of Coagulase-negative staphylococci (CoNS) and $80 \%(28 / 35)$ of S. aureus. Significant methicillin resistance rate was identified in $S$. aureus which was $88.2 \%$
(15/17) (Additional file 1: supplement I). All the isolates of CoNS, Staphylococcus aureus and GBS were susceptible to vancomycin, but three enterococci isolates $(16.7 \%, 3 / 18)$ in HALOS were resistant which were from three different NICUs. 
For Fungi, 30.4\% (28/92) were detected from maternal and child health hospital and 69.6\% (64/92) from general hospital. 97.8\% (90/92) of Fungi cases were identified in HALOS. Candida albicans (42.4\%; 39/92) and Candida parapsilosis $(19.6 \%$; $18 / 92)$ were the top two species isolated (Table 2). All Fungi were sensitive to 5-fluorocytosine, amphotericin B and voriconazole. Candida albicans strains were resistant to both fluconazole $(3.3 \%$; $3 / 92)$ and itraconazole $(3.3 \% ; 3 / 92)$. Fungal BSI occurred in 28 of 405 infants in 8 NICUs (6.9\%) with routine use of antifungal prophylaxis, whereas 64 in 683 infants in the other 17 NICUs $(9.4 \%)$ with using antifungal drugs only in necessity. No significant difference were observed on fungal BSI based on whether or not routine use of antifungal prophylaxis in NICUs $(9.4 \%$ vs $6.9 \%$, [OR] 0.718, 95\% CI 0.452-1.140; $P=0.161$ ).

\section{Community-acquired LOS}

In CALOS, 97.6\% (40/41) of pathogens were detected from neonates with normal birth weight and 90.2\% (37/41) from term infants (Table 1). In contrast, $87.8 \%$ $(36 / 41)$ of pathogens that caused CALOS were GP bacteria. GBS and Staphylococcus aureus were responsible for $46.3 \%(19 / 41)$ and $41.5 \%$ (17/41) of CALOS cases. None of the five GN pathogens causing CALOS were carbapenem and multidrug resistant. High rates of resistance were observed in GBS in CALOS to erythromycin $(89.5 \%, 17 / 19)$ and clindamycin $(80 \%, 15 / 19)$ (Additional file 1: supplement I).

\section{Differences in neonatal period and beyond}

The majority of pathogens were identified during 28 days of life $(84.3 \%, 921 / 1092)$. The most common pathogens during 28 days of life were Escherichia coli (21.7\%, 200/921), Klebsiella pneumoniae (19\%, 175/921) and CoNS (9.1, 84/921). In preterm, Klebsiella pneumoniae (30.4\%, 52/171), Fungi $(18.1 \%, 31 / 171)$ and CoNS $(14.6 \%$, 25/171) were the top three pathogens after 28 days of life in hospitalization. Carbapenem resistance rates in Klebsiella pneumoniae were $12 \%(21 / 175)$ and 9.6\% (5/52), and multidrug resistance rates $56.6 \%(99 / 175)$ and $42.3 \%$ (22/52) during 28 days of life and after 28 days of life in hospitalization, respectively. Five carbapenem resistant Escherichia coli and vancomycin resistant Enterococcus spp. were all identified during 28 days of life. No difference was found about all-cause mortality during 28 days of life and after 28 days of life in hospitalization (5.7\% vs 2.9\%, [OR] 0.505, 95\% CI 0.199-1.282; $P=0.151$ ).

\section{Discussion}

Due to different empirical uses of antibiotics and implementation of preventive measures, the pathogens involved in neonatal BSI may vary geographically and temporally [9]. The results state that Escherichia coli, Klebsiella pneumoniae and GBS were the leading causes, respectively. This study was conducted in China, a lowermiddle income country with paucity of high-quality data. The most common organism causing HALOS was Klebsiella pneumoniae and approximately $2 / 3$ of those isolates were MDR or resistance of third-generation cephalosporin. The result would be important for clinicians in Chinese NICUs to guide optimal clinical prevention strategies and for consideration of empirical antibiotic treatment during clinical management.

The proportion of all positive blood cultures judged to be contaminants in our study was nearly $60 \%$ which was much higher than the proportion of $13-56 \%$ reported from western countries [25-27]. Reports from 12 British hospitals revealed approximately half blood culture judged to contaminants in 5 hospitals compared with no more than a quarter in the other 7 hospitals [28]. This is striking, nevertheless, few relevant data was reported in China. Standardizing blood culture collection methods, optimizing blood volume, creating checklists, and reinforcing nurse education were verified to develop a best practice for reduction of blood culture contamination [29].

Nosocomial infection is a major health problem particularly in NICUs in developing countries [30]. In current study, about two thirds of pathogens identified were responsible for HALOS. Approximately $60 \%$ of HALOS were due to $\mathrm{GN}$ bacteria which was similar to the results from previous Chinese studies [11, 12]. A review of 11,471 bloodstream samples indicated that GN bacteria was detected from no less than $60 \%$ of positive blood cultures in all the developing settings of the world [31]. Similar to that reported from South Asia [3] and Egypt [7], Klebsiella pneumoniae was the most common GN bacteria causing LOS. By contrast, CoNS was the most common pathogen in western countries for LOS, such as $40 \%$ in Switzerland [14]. The preponderance of CoNS might indicate the developed regions' adoption of neonates with lower gestational age and lower birth weight and prolonged use of central catheters which are risk factors for CoNS infection [32]. The predominance of GN bacteria in our developing counties may largely be attributed to the lack of standard infection-control practices. Insufficient hand hygiene, lack of essential equipment and supplies including sinks, running water and disposables, overcrowding and understaffing are described to be key contributors to nosocomial infection caused by GN bacteria [33]. A recent prospective population-based cohort study reported HALOS frequently correlated with low gestational age, low birth weight and comorbidities. The study population in our cohort appears similar to that previously reported [14]. Therefore, implementation 
of these basic hygiene practices should be emphasized more in Chinese NICUs to minimize the hazards of the high incidence of HALOS caused by GN bacteria. Also, empiric antibiotics selected to treat suspected HALOS in Chinese NICUs need to effectively treat GN bacteria, especially Klebsiella pneumoniae.

Differences in all-cause mortality between EOS and HALOS may be partially explained by the prophylactically antibiotics prescribed and unrestricted use of broad-spectrum and advanced antibiotics in our NICUs, although it is highly discouraged. For part of neonates diagnosed with EOS, born with shock, disseminated intravascular coagulation and multiple organ failure, they had poor outcomes frequently even with active treatment.

In the current study, no neonate experienced both EOS and HALOS. This may be due to the fact that infants with EOS were less likely to be of low birth weight and lower gestational age, which may have resulted in them being discharged after a short course of antibiotics, avoiding an excessive length of stay and prolonged hospital exposure and interventions.

Another remarkable finding in the Chinese NICUs was the relatively high percentage of Fungi in HALOS. Nearly all the identified Fungi infections were responsible for HALOS, which was paralleled with other Chinese studies $[11,12]$. Similarly, recent studies have also reported outbreaks of fungal nosocomial infection in Chinese NICUs $[34,35]$. In China, huge variation existed among NICUs in the use of antifungal prophylaxis. In a recent multicenter study, antifungal drugs were prescribed in $20 \%$ of LOS and 35\% of fungal LOS, including prophylaxis or empirical treatment [12]. Prolonged antibiotic therapy, broad spectrum antibiotic exposure may be the connection to high prevalence of fungal nosocomial infections [36]. This highlights the need to develop new and more effective approaches to prevent HALOS.

A strength of this study was that all 25 hospitals have their own maternity/obstetric ward and almost all neonates were born onsite with only a few transferred. This makes the pathogens in EOS more precise and representative. In the current study, Escherichia coli was the most common pathogen in EOS, followed by GBS. Similarly, the latest surveillance from a national neonatal research network in US demonstrated the shift from GBS to E. coli as the leading pathogen and the increase in Escherichia coli infections among very low-birth-weight infants [37]. Escherichia coli was the most common pathogen among preterm infants in current study which was similar to those reported in most developed countries [6, 14, 37]. Previously, GBS was reported to be a rare cause of EOS and was documented in only a few reports from China and other Asian countries [7, 38]. The reason for the low proportion of GBS in EOS may be partially due to the overuse of antenatal antibiotics in China. However, we did not have the data on antenatal antibiotic use. Invasive GBS infections in Chinese neonates are supposed to be rare because of the lower rate of $G B S$ colonization in pregnant women and the higher protective antibody concentrations in mothers, so that screening and preventive measurements have been suspended [38]. Escherichia coli was the most common pathogen in EOS and the second common in HALOS. The extraordinary similarity of this spectrum supports the assumption that the cause of EOS may not only be due to vertical transmission from mothers but also can be caused by unsanitary practices in the labour rooms and NICUs. Further investigations are of urgent need to identify the causes of GN bacteria in EOS in China and subsequently develop targeted prevention strategies.

Most CALOS occurred in term or near-term newborns, accounting for a small percentage of BSI. GBS and Staphylococcus aureus were the leading responsible pathogens. Similar to reports in developed counties, infants with CALOS had a higher birth weight and gestational age and fewer adverse outcomes compared to those who developed HALOS [14]. The relatively low incidence rate may be related to the lack of strict management and relatively easy access to antibiotics in China. Antibiotics may have been administered before admission resulting in false negative results in blood cultures.

Our study showed that isolation frequency and AMR of GN bacteria differed significantly between EOS and HALOS. Bacteria strains isolated in HALOS were more resistant to ampicillin, gentamicin, third-generation cephalosporin and carbapenem evaluated in our study compared to those isolated in EOS. Studies from both developing and developed countries have also shown similar findings $[3,20]$. This may suggest that bacteria strains associated with vertical transmission are different to those that are nosocomial transmission and supports the idea that different antimicrobial regimens are needed.

In our study, a concerning feature of the HALOS pathogens is the high resistance rates in Klebsiella pneumoniae to the third-generation cephalosporins and carbapenems, and the proportion of multi-drug resistant strains. Notably a multi centre South Asia study involving nosocomial infections reported resistance rates in Klebsiella pneumoniae of $71.3-73.7 \%$ to cefotaxime and $9.4-11.5 \%$ to meropenem. Multidrug resistance was reported in 66.1$75.3 \%$ of Klebsiella pneumoniae [3]. In a Taiwanese study, the overall proportion of GN infection was comparable to that in our study, and K. pneumoniae was the most common isolate, but the rate of carbapenem resistance (18.6\%) was higher than our rate [39]. Also, they found that the most frequent mechanism of MDR GN bacteria 
was ESBL production [39]. Ampicillin/piperacillin and third-generation cephalosporin are the first choices for empirical treatment to neonatal sepsis in China [40]. Unrestricted use of broad-spectrum cephalosporin may explain the high drug resistance rates in Klebsiella pneumoniae and makes the choice of antibiotics extremely difficult.

In general, the severe situation of high antimicrobial resistance rate in HALOS is probably multifactorial and may include lack of standardized infection control policies, higher rates of broad-spectrum antibiotics use and the low nurse-to-bed ratios observed in NICUs.

Strengths of this study include its enrollment of pathogens from all neonates admitted to the NICU, distinguishing pathogens and AMR between EOS, HALOS and CALOS, and large sample size. The following limitations should be considered: (1) the choice of a definition on neonatal sepsis is a limitation inherent to many studies. The diagnosis of neonatal sepsis is mainly based on Chinese Consensus formulated by Chinese Pediatric Society which may not have been widely accepted [30]. The diagnosis of infection of CONS usually relies on confirmation with second blood culture, but this practice is not routinely followed in LMICs. Therefore we are not certain whether CONS represented true pathogen infections or potential contaminants; (2) antibiotics are usually administered to neonates born offsite with suspected sepsis, which could impair recovery of pathogens associated with CALOS. Neonates who were discharged and present with CALOS at a later stage are not necessarily admitted to the tertiary facility where they were born, and as such, may have been omitted from this study; (3) we merely collected the data on the proportion of causative pathogens between EOS, HALOS and CALOS, but we were unable to calculate the incidence rates.

\section{Conclusion}

In this study, EOS and HALOS were most commonly caused by GN bacteria, with Escherichia coli and Klebsiella pneumoniae being the major pathogens, respectively. Only a small proportion of pathogens were identified for CALOS, most commonly GBS. A high proportion of the pathogens isolated were due to HALOS, and the prevalence of AMR was high. Effective interventions are urgently needed to reduce HALOS in LMICs. Continued surveillance is warranted to identify pathogen distribution and AMR, and to distinguish between EOS, HALOS and CALOS causing agents.

\section{Abbreviations}

BSI: Neonatal bloodstream infection; AMR: Antimicrobial resistance; GBS: Group B streptococcus; EOS: Early-onset sepsis; HALOS: Hospital-acquired late-onset sepsis; CALOS: Community-acquired late-onset sepsis; GN: Gram negative; GP: Gram positive; LMICs: Low-income and middle-income countries; CONS: Coagulase negative staphylococcus; MDR: Multidrug-resistant; AST: Antimicrobial susceptibility testing.

\section{Supplementary Information}

The online version contains supplementary material available at https://doi. org/10.1186/s13756-021-00989-6.

Additional file 1. Antimicrobial Resistance of Common EOS and HALOS Causing Pathogens at 25 NICUs, January 2017 - December 2019.

\section{Acknowledgements}

We would like to thank Zhang-bin Yu from Nanjing Maternal and Child Health Hospital of Nanjing Medical University for assistance with this research project.

\section{Authors' contributions}

YHY, the corresponding author, doctorate, and professor of medicine, designed the study, trained and supervised the data collectors, interpreted the results and revised the manuscript. The first author, namely, $J L$, played a role in the analysis and interpretation of the data and in the preparation and drafting of the manuscript. The co-first authors, participated in the design of the study, the collection and interpretation of the data and writing of the manuscript. All authors listed on the manuscript approved the submission of this version of the manuscript and would accept full responsibility for the manuscript. All authors read and approved the final manuscript.

\section{Funding}

This work was supported by Key Research and Development Program of Shandong (Grant No. 2018GSF118163) and Shandong Provincial Medical Health Technology Development Project (Grant No. 2017WS009).

\section{Availability of data and materials}

The data that support the findings of this study are available from the corresponding authors upon reasonable request.

\section{Declarations}

Ethics approval and consent to participate

The Institutional Review Board of Shandong Provincial Hospital Affiliated with Shandong University approved this project. All authors have signed written informed consent and approved the submission of this version of the manuscript and take full responsibility for the manuscript. The legal guardian of all participants signed an informed consent form that their data could be used for various clinical studies.

\section{Author details}

${ }^{1}$ Department of Neonatology, Shandong Provincial Hospital Affiliated to Shandong First Medical University, Jinan 250021, Shandong, China. ${ }^{2}$ Department of Neonatology, Shandong Provincial Hospital, Cheeloo College of Medicine, Shandong University, No. 234, Jingwu Road, Huai Yin District, Jinan 250021, Shandong, China. ${ }^{3}$ Department of Pediatrics, Yantai Yuhuangding Hospital, Yantai 264000, Shandong, China. ${ }^{4}$ Department of Neonatology, The First Affiliated Hospital of Shandong First Medical University, Jinan, China. ${ }^{5}$ Department of Neonatology, Weifang Maternal and Child Health Hospital, Weifang, China. ${ }^{6}$ Department of Pediatrics, Baogang Third Hospital of Hongci Group, Baotou, China. ${ }^{7}$ Department of Neonatology, Zibo Maternal and Child Health Hospital, Zibo, China. ${ }^{8}$ Department of Pediatrics, People's Hospital of Rizhao, Rizhao, China. ${ }^{9}$ Department of Pediatrics, Binzhou Medical University Hospital, Binzhou, China. ${ }^{10}$ Department of Pediatrics, Linyi People's Hospital, Linyi, China. ${ }^{11}$ Department of Pediatrics, Women and Children's Health Care Hospital of Linyi, Linyi, China. ${ }^{12}$ Department of Pediatrics, Weifang Yidu Central Hospital, Weifang, China. ${ }^{13}$ Department of Neonatology, Jinan Maternity and Child Health Care Hospital, Jinan, China. ${ }^{14}$ Department of Pediatrics, Dongying People's Hospital, Dongying, China. ${ }^{15}$ Department of Pediatrics, Tengzhou Central People's Hospital, Tengzhou, China. ${ }^{16}$ Department of Pediatrics, Hebei Petro China Central Hospital, Langfang, China. ${ }^{17}$ Department of Neonatology, Shandong Provincial Maternity and Child Health Care Hospital, Jinan, 
China. ${ }^{18}$ Department of Pediatrics, The Second Affiliated Hospital of Shandong First Medical University, Taian, China. ${ }^{19}$ Department of Pediatrics, Jinan Central Hospital, Cheeloo College of Medicine, Shandong University, Jinan, China. ${ }^{20}$ Department of Pediatrics, Shandong Provincial Hospital Affiliated to Shandong First Medical University, Jinan, China. ${ }^{21}$ Department of Pediatrics, Dezhou People's Hospital, Dezhou, China. ${ }^{22}$ Department of Pediatrics, Zibo Central Hospital, Zibo, China. ${ }^{23}$ Department of Pediatrics, Liaocheng People's Hospital, Liaocheng, China. ${ }^{24}$ Department of Pediatrics, The Second People's Hospital of Liaocheng, Liaocheng, China. ${ }^{25}$ Department of Neonatology, The Second Children and Women's Healthcare of Jinan City, Jinan, China. ${ }^{26}$ Department of Pediatrics, Heze Municipal Hospital, Heze, China.

\section{Received: 5 January 2021 Accepted: 1 August 2021}

\section{Published online: 16 August 2021}

\section{References}

1. Liu L, Oza S, Hogan D, Perin J, Rudan I, Lawn JE, et al. Global, regional, and national causes of child mortality in 2000-13, with projections to inform post-2015 priorities: an updated systematic analysis. The Lancet. 2015;385(9966):430-40.

2. Naghavi M, Abajobir AA, Abbafati C, Abbas KM, Abd-Allah F, Abera $S F$, et al. Global, regional, and national age-sex specific mortality for 264 causes of death, 1980-2016: a systematic analysis for the Global Burden of Disease Study 2016. The Lancet. 2017;390(10100):1151-210.

3. Chaurasia S, Sivanandan S, Agarwal R, Ellis S, Sharland M, Sankar MJ. Neonatal sepsis in South Asia: huge burden and spiralling antimicrobial resistance. BMJ. 2019;364:k5314.

4. Folgori L, Ellis SJ, Bielicki JA, Heath PT, Sharland M, Balasegaram M. Tackling antimicrobial resistance in neonatal sepsis. Lancet Glob Health. 2017;5(11):e1066-8.

5. Chereau F, Opatowski L, Tourdjman M, Vong S. Risk assessment for antibiotic resistance in South East Asia. BMJ. 2017;358:j3393.

6. Hongsuwan M, Srisamang P, Kanoksil M, Luangasanatip N, Jatapai A, Day NP, et al. Increasing incidence of hospital-acquired and healthcareassociated bacteremia in northeast Thailand: a multicenter surveillance study. PLoS ONE. 2014;9(10):e109324.

7. Mohsen L, Ramy N, Saied D, Akmal D, Salama N, Abdel Haleim MM, et al. Emerging antimicrobial resistance in early and late-onset neonatal sepsis. Antimicrob Resist Infect Control. 2017;6:63.

8. Gkentzi D, Kortsalioudaki C, Cailes BC, Zaoutis T, Kopsidas J, Tsolia M, et al. Epidemiology of infections and antimicrobial use in Greek Neonatal Units. Arch Dis Child Fetal Neonatal Ed. 2019;104(3):F293-7.

9. Schrag SJ, Farley MM, Petit S, Reingold A, Weston EJ, Pondo T, et al. Epidemiology of invasive early-onset neonatal sepsis, 2005 to 2014 Pediatrics. 2016;138(6):e20162013. https://doi.org/10.1542/peds. 2016-2013.

10. Li JY, Chen SQ, Yan YY, Hu YY, Wei J, Wu QP, et al. Identification and antimicrobial resistance of pathogens in neonatal septicemia in China-a meta-analysis. Int J Infect Dis. 2018;71:89-93.

11. Gao K, Fu J, Guan X, Zhu S, Zeng L, Xu X, et al. Incidence, bacterial profiles, and antimicrobial resistance of culture-proven neonatal sepsis In South China. Infect Drug Resist. 2019;12:3797-805.

12. Jiang S, Yang C, Yang C, Yan W, Shah V, Shah PS, et al. Epidemiology and microbiology of late-onset sepsis among preterm infants in China, 2015-2018: a cohort study. Int J Infect Dis. 2020;96:1-9.

13. Jiang S, Hong L, Gai J, Shi J, Yang Y, Lee SK, et al. Early-onset sepsis among preterm neonates in China, 2015 to 2018. Pediatr Infect Dis J. 2019;38(12):1236-41.

14. Giannoni E, Agyeman PKA, Stocker M, Posfay-Barbe KM, Heininger U, Spycher BD, et al. Neonatal sepsis of early onset, and hospital-acquired and community-acquired late onset: a prospective population-based cohort study. J Pediatr. 2018;201:106-114 e4.

15. Clinical and Laboratory Standards Institute. Performance Standards for Antimicrobial Susceptibility Testing: Nineteenth Informational Supplement. Wayne, PA: CLSI. 2017.
16. Clinical and Laboratory Standards Institute. Performance Standards for Antimicrobial Susceptibility Testing: Nineteenth Informational Supplement. Wayne, PA: CLSI. 2018.

17. Fitchett EJA, Seale AC, Vergnano S, Sharland M, Heath PT, Saha SK, et al. Strengthening the reporting of observational studies in epidemiology for newborn infection (STROBE-NI): an extension of the STROBE statement for neonatal infection research. Lancet Infect Dis. 2016;16(10):e202-13.

18. Shane AL, Sánchez PJ, Stoll BJ. Neonatal sepsis. Lancet. 2017;390(10104):1770-80.

19. Chinese Pediatric Society. Chinese consensus of diagnosis and treatment of neonatal sepsis. Chin J Peditr. 2003;41(12):897-9.

20. Cailes B, Kortsalioudaki C, Buttery J, Pattnayak S, Greenough A, Matthes J, et al. Epidemiology of UK neonatal infections: the neon IN infection surveillance network. Arch Dis Child Fetal Neonatal Ed. 2018;103(6):F547-53.

21. Chinese Pediatric Society. Chinese consensus of diagnosis and treatment of neonatal sepsis. Chin J Peditr. 2019;57(4):252-7.

22. Cailes B, Kortsalioudaki C, Buttery J, Pattnayak S, Greenough A, Matthes J, et al. Antimicrobial resistance in UK neonatal units: neonIN infection surveillance network. Arch Dis Child Fetal Neonatal Ed. 2018;103(5):F474-8.

23. Magiorakos AP, Srinivasan A, Carey RB, et al. Multidrug-resistant, extensively drug-resistant and pandrug-resistant bacteria: an international expert proposal for interim standard definitions for acquired resistance. Clin Microbiol Infect. 2012;18(3):268-81.

24. Johnson J, Robinson ML, Rajput UC, Valvi C, Kinikar A, Parikh TB, et al. High burden of bloodstream infections associated with antimicrobial resistance and mortality in the neonatal intensive care unit in Pune, India. Clin Infect Dis. 2021;73(2):271-280. https://doi.org/10.1093/cid/ ciaa554.

25. Washer LL, Chenoweth C, Kim HW, et al. Blood culture contamination: a randomized trial evaluating the comparative effectiveness of 3 skin antiseptic interventions. Infect Control Hosp Epidemiol. 2013;34(1):15-21.

26. Dargère $\mathrm{S}, \mathrm{Cormier} \mathrm{H}$, Verdon R. Contaminants in blood cultures: importance, implications, interpretation and prevention. Clin Microbiol Infect. 2018;24(9):964-9.

27. Doern GV, Carroll KC, Diekema DJ, et al. Practical guidance for clinical microbiology laboratories: a comprehensive update on the problem of blood culture contamination and a discussion of methods for addressing the problem. Clin Microbiol Rev. 2019;33(1):e00009-19.

28. Harvey DJ, Albert S. Standardized definition of contamination and evidence-based target necessary for high-quality blood culture contamination rate audit. J Hosp Infect. 2013;83(3):265-6.

29. El Feghaly RE, Chatterjee J, Dowdy K, et al. A Quality improvement initiative: reducing blood culture contamination in a Children's Hospital. Pediatrics. 2018;142(4):e20180244.

30. Abdel-Wahab F, Ghoneim M, Khashaba M, El-Gilany AH, Abdel-Hady D. Nosocomial infection surveillance in an Egyptian neonatal intensive care unit. J Hosp Infect. 2013;83(3):196-9.

31. Zaidi AKM, Huskins WC, Thaver D, Bhutta ZA, Abbas Z, Goldmann DA. Hospital-acquired neonatal infections in developing countries. The Lancet. 2005;365(9465):1175-88.

32. Jean-Baptiste N, Benjamin DK Jr, Cohen-Wolkowiez M, Fowler VG Jr, Laughon M, Clark RH, et al. Coagulase-negative staphylococca infections in the neonatal intensive care unit. Infect Control Hosp Epidemiol. 2011;32(7):679-86.

33. Von Dolinger de Brito $D$, de Almeida Silva $H$, Jose Oliveira $E$, Arantes A, Abdallah VO, Tannus Jorge M, et al. Effect of neonatal intensive care unit environment on the incidence of hospital-acquired infection in neonates. J Hosp Infect. 2007;65(4):314-8.

34. Fu J, Ding Y, Wei B, Wang $L$, Xu S, Qin P, et al. Epidemiology of Candida albicans and non-C. albicans of neonatal candidemia at a tertiary care hospital in western China. BMC Infect Dis. 2017;17(1):329.

35. Fu J, Wang $X$, Wei $B$, Jiang $Y$, Chen J. Risk factors and clinical analysis of candidemia in very-low-birth-weight neonates. Am J Infect Control. 2016:44(11):1321-5. 
36. Aliaga S, Clark RH, Laughon M, Walsh TJ, Hope WW, Benjamin DK, et al. Changes in the incidence of candidiasis in neonatal intensive care units. Pediatrics. 2014;133(2):236-42.

37. Stoll BJ, Puopolo KM, Hansen NI, Sanchez PJ, Bell EF, Carlo WA, et al. Early-onset neonatal sepsis 2015 to 2017, the rise of Escherichia coli, and the need for novel prevention strategies. JAMA Pediatr. 2020;174(7):e200593.

38. Russell NJ, Seale AC, O'Driscoll M, O'Sullivan C, Bianchi-Jassir F, Gonzalez-Guarin J, et al. Maternal Colonization With Group B Streptococcus and Serotype Distribution Worldwide: Systematic Review and Meta-analyses. Clin Infect Dis. 2017;65(suppl_2):S100-S11. https://doi. org/10.1093/cid/cix658
39. Tsai MH, Chu SM, Hsu JF, Lien R, Huang HR, Chiang MC, et al. Risk factors and outcomes for multidrug-resistant Gram-negative bacteremia in the NICU. Pediatrics. 2014;133(2):e322-9.

40. Dong Y, Jiang SY, Zhou Q, Cao Y. Group B Streptococcus causes severe sepsis in term neonates: 8 years experience of a major Chinese neonatal unit. World J Pediatr. 2017;13(4):314-20.

\section{Publisher's Note}

Springer Nature remains neutral with regard to jurisdictional claims in published maps and institutional affiliations.
Ready to submit your research? Choose BMC and benefit from:

- fast, convenient online submission

- thorough peer review by experienced researchers in your field

- rapid publication on acceptance

- support for research data, including large and complex data types

- gold Open Access which fosters wider collaboration and increased citations

- maximum visibility for your research: over 100M website views per year

At BMC, research is always in progress.

Learn more biomedcentral.com/submissions 